\title{
The Degree of Bone Mineral Density Improvement in Patients with Osteoporosis on Current NICE Recommended Treatment: An Audit
}

\section{Rachel Reed, Parijat De}

University of Birmingham and Department of Diabetes, Endocrinology \& Lipid Metabolism, City Hospital, Birmingham B18 7QH

\section{Background}

- Osteoporosis is a progressive systemic skeletal disease characterised by reduced bone mineral density and micro-architectural deterioration of bone tissue. 'In England and Wales over 2 million people have osteoporosis.2

- Over 300,000 patients present to UK hospitals with fragility fractures each year.

- 2 million bed days are a result of osteoporotic fractures. Hip fractures are associated with increased mortality.

- Prevalence of osteoporosis is more than $25 \%$ in 80 year olds and with people living longer, it is essential that patients are treated effectively with evidence based treatments.

- The major consequences of osteoporosis are increased bone fragility and risk of fracture. ${ }^{2}$

\section{Aims}

- To identify whether current osteoporosis treatment is NICE recommended

- To identify improvements in bone mineral density (BMD) in patients with osteoporosis on NICE recommended treatment

- To check treatment compliance

- To identify whether patients with osteoporosis are taking calcium and vitamin D supplements

\section{DEXA SCAN}

- $\quad T$ - score: the number of standard deviations (SD) below the mean BMD of young adults at their peak bone mass

- Osteoporosis is defined as a T-score of -2.5 SD or below on DEXA scanning

- Severe Osteoporosis: a T - score of -2.5 SD or below on DEXA scanning PLUS fragility fracture

- Osteopenia: a T - score between -1 and -2.5 on DEXA scanning

- Normal: $T$ - score more than or equal to -1

- DEXA scan should be done if two of these are found : maternal hip fracture, cigarette smoker, height loss of $>2 \mathrm{~cm}$, low body weight and low BMD by any method other than DEXA

\section{NICE Risk Factors For Osteoporosis}

Independent clinical risk factors for fracture

- Parental history of hip fracture

- Alcohol intake of 4 or more units per day

- Rheumatoid arthritis

Indicators of low BMD

- $\quad$ Low $B M I<22$

- Ankylosing spondylitis

- Crohn's disease

- Conditions resulting in prolonged mobility

\begin{tabular}{|ll|}
\hline & NICE Guided Treatment \\
\hline $\begin{array}{l}\text { Bisphosphonates } \\
\text { Modulator }\end{array}$ & Alenronate, etidronate, risedronate \\
Strontium ranelate & Raloxifene \\
& Similar properties to calcium (increases \\
Teriparetide & bone formation and reduced resorption) \\
\hline
\end{tabular}

\section{Audit Standards and Criteria}

- Our standards are based on the national and local guidelines:

"Primary and secondary prevention of osteoporotic fragility fractures in post menopausal women" NICE guidance 20111

- "SWBH medical management of men and women who are at risk of osteoporosis highlighting management of glucocorticoid induced osteoporosis" 3

- Patients should have had at least 2 DEXA scan results (before and after at least 1 year of treatment)

- $\quad$ Patients should have or have previously had diagnosed osteoporosis

- The patients should be taking calcium and vitamin

- The patient should be compliant with treatment

\section{Methods \& Materials}

- A retrospective audit was carried out at Birmingham City Hospital between June 2011 October 2012

- Patient information from 31 patients was gathered from an electronic data base (Clinica Data Archive) and clinic notes

- 24 out of the 31 patients $(77 \%)$ met the audit standards

- To assess Improvement in bone mineral density we looked at DEXA scan T - scores results we compared 2 DEXA results, before and after at least one year of treatment

- We used a data collection sheet to record information on: patient age, gender, ethnicity, diagnosed osteoporosis (Y/N), duration of osteoporosis, current treatment and compliance, any improvement in BMD, first mean hip and spine DEXA T - score, last DEXA T - score, risk factors for osteoporosis, and number of years follow up since diagnosis

\section{Results}

More than half of the patients (58\%) had an improvement in their BMD on NICE guided treatment (Figure 1). $83 \%$ had diagnosed osteoporosis and 17\% had improved their T-scores enough to become osteopenic. All of the patients with osteoporosis were taking calcium and vitamin $\mathrm{D}$ along with three quarters of the osteopenic patients. The only patient not taking calcium and vitamin D stopped treatment after a parathyroidectomy operation for an adenoma.

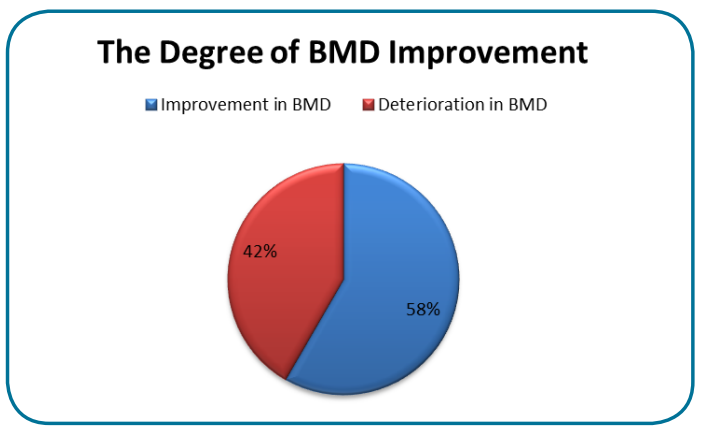

Feople had an improvement in BMD in both the spine and femur T- scores spine and femur $T$ - scores improvement: 0.44 SD

provement: 1.95 SD Orenti - scose improveOverall - score im ment :1.2 SD

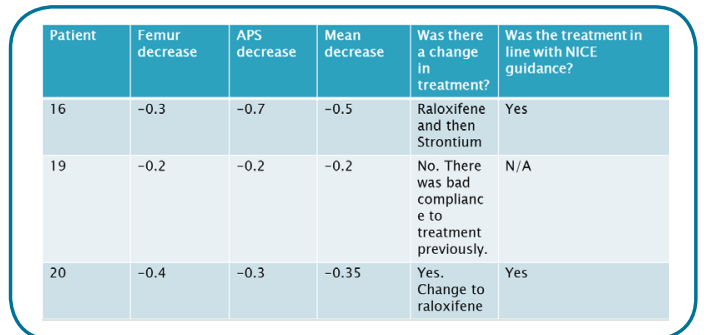

Figure 2

3 patients had a deterioration in spinal and femur T-score

Patient Risk Factors

- Independent clinical risk factor: one patient has rheumatoid arthritis and was on long term systemic glucocorticoids

- Indicators of low BMD: ankylosing spondylitis (1), prolonged immobility (2) and ulcerative colitis (2)

\begin{tabular}{l}
\hline Patients with Risk Factors Associated with Secondary Osteoporosis \\
\hline Klinefelter's syndrome (1) \\
Hypothyroidism (5) \\
Hyperparathyroidism (4) \\
Parathyroid adenoma (2) \\
Hypogonadism (5) \\
Sickle cell and Thalassemia (4) \\
Hypoparathyroid (1) \\
Others: raised ALP and PTH (incidental) \\
\hline
\end{tabular}

The Mean age of patients was 55 years and the mean durations of osteoporosis follow up was 4 years. The majority of patients were female $(63 \%)$. Treatment compliance was difficult to evaluate as it has not been documented in the majority of the notes but some patients forgot to take their vitamin $\mathrm{D}$.

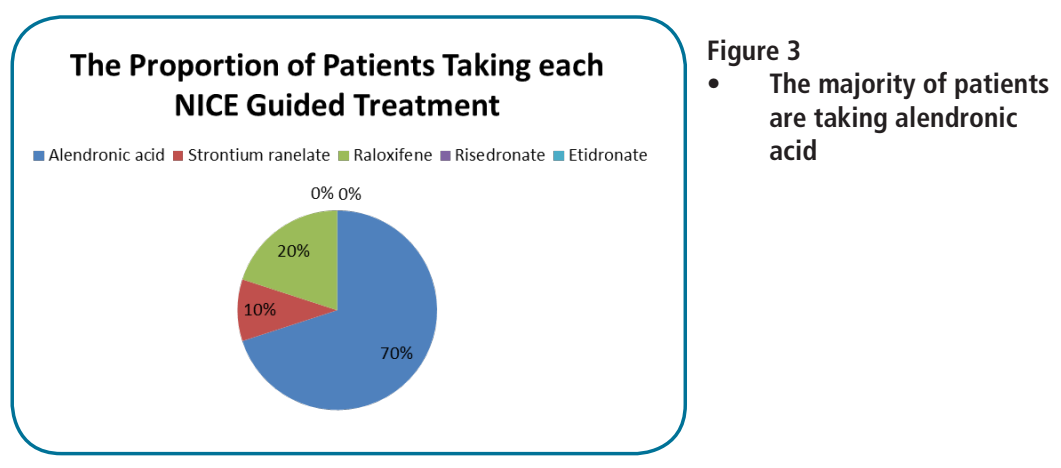

\section{Conclusions and Recommendations}

- Osteoporosis is an important endocrine problem with significant morbidity and mortality and there are effective current NICE guided treatments strategies available

- The main duration of osteoporosis follow up was 4 years and the mean age of patients was 55. The majority of participants were white Caucasian females

- $70 \%$ of patients were taking alendronic acid and all osteoporotic patient were taking vitamin $\mathrm{D}$ and calcium

- There was an improvement in BMD of patients with osteoporosis on NICE recommended treatment in this current audit - 58\% had an Improvement in both femur and mean APS T scores with a mean improvement of $1.2 \mathrm{SD}$

- Most patients adhere to their drugs although compliance was difficult to ascertain from the data base.

- In future we plan to audit compliance and patient satisfaction prospectively

\section{References}

- 1: Primary and Secondary Prevention of Osteoporotic fragility fractures in postmenopausal women. Implementing NICE guidance: NICE technology appraisal guidance 160 and 151; 2008

- 2: Osteoporosis: fragility fracture risk. Short clinical guideline-CG146: Evidence and recommendations; August 2012

- Medical Management of women and men who are at risk of osteoporosis highlighting management of glucocorticoid -induced osteoporosis: SWBH treatment guidelines 